\title{
Exploration and Practice of College Mathematics Teaching Based on STEM Education Concept
}

\author{
Fan Qingduan ${ }^{1 \mathrm{a}}$, Zheng Zhongtuan ${ }^{1 \mathrm{~b}}$, Wang Guoqiang ${ }^{1 \mathrm{c}^{*}}$ \\ ${ }^{1}$ School of Mathematics, Physics and Statistics, Shanghai University of Engineering Science, Shanghai, China \\ afanqingduan@163.com, ${ }^{b}$ zhongtuanzheng@163.com, ${ }^{c}$ guoq_wang@hotmail.com \\ *Corresponding author
}

\begin{abstract}
STEM education is a hot topic in the current research field of education research and reform. Based on the background and the development of the STEM education at home and abroad, this paper summarizes and analyzes the main characteristics and the practice forms of university STEM education, and gives the beneficial enlightenment of the STEM concept for the reform of college mathematics teaching and education. From the aspects of courses and projects, integration of industry, teaching and research, the specific exploration and practice of mathematics teaching in our university based on the STEM education concept are introduced. The practice shows that the STEM education concept is helpful to promote the reform of college mathematics teaching mode to cultivate students' innovative ability and innovative thinking, and promote the implementation of the outstanding engineer training plan and the construction of new engineering subjects.
\end{abstract}

Keywords: STEM, college mathematics teaching, integration of industry and teaching, integration of industry and research

\section{基于 STEM 教育理念的大学数学教学探索与实践}

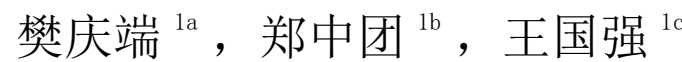

${ }^{1}$ 上海工程技术大学数理与统计学院, 上海, 中国

afanqingduan@163.com, ${ }^{b}$ zhongtuanzheng@163.com, ${ }^{* *}$ guoq_wang@hotmail.com *通讯作者

\section{摘要}

STEM 教育是当前国际教育研究和改革的热点问题。在 STEM 教育的背景与国内外 STEM 教育的发展基础上, 总 结分析了大学 STEM 教育的主要特征与开展形式, 给出了 STEM 理念对于大学数学教学教育改革的有益启示。从 课程与项目、产教融合与产研融合等方面, 介绍了我校大学数学教学基于 STEM 教育理念的具体探索与实践。 实践表明, STEM 教育理念有助于推动革新大学数学教学模式进而培养学生创新能力与创新思维, 促进卓越工 程师培养计划实施与新工科建设。

关键词: STEM，大学数学教学，产教融合，产研融合

\section{1. 前言}

STEM 是科学 (Science)、技术 (Technology)、工 程 (Engineering) 和数学 (Mathematics) 等学科的简 称。国际竞争力依靠的是科技创新和人才培养，维持 世界科技强国的地位要改革教育体制。早在 1986 年, 美国国家科学委员会发布的《本科科学、数学和工程
教育报告》首次明确提出科学、数学、工程和技术教 育的大融合，强调多学科的交叉融合与有机结合，被 视为 STEM 教育的开端 ${ }^{[1]}$ 。随之许多国家实施了 STEM 教育, 美国学者 G. Yakman 主张将人文艺术 (Art) 融入 STEM 教育，于 2006 年提出 STEAM 教育。

虽然美国的高等教育取得了丰硕成绩, 然而, 据 统计美国读 STEM 相关专业的本土学生也在减少，还 
有许多国家出现了技术性人才缺乏、毕业生知识结构 不合理以及创新能力不足等问题 ${ }^{[2]}$ 。究其原因, 以学 科模式传授知识的教学模式尽管易于分工、便于实施, 但是课堂上研究的问题缺乏问题的真实性与趣味性, 不利于培养学生的探索精神等弊端越来越明显。针对 这些突出问题和社会需求, 高等教育涌现出了打破学 科、整合学科教育等人才培养理念 ${ }^{[3]}$ 。2012 年 2 月, 美国总统科技顾问委员会发表了《Engage to Exce1: Producing One Million Additional College Graduates with Degrees in Science, Technology, Engineering and Mathematics》, 使得美国的本科 STEM 教育得到了明显的进步 ${ }^{[4]}$ 。2018 年美国发布了 21 世纪研究生 STEM 教育报告与美国发布的《制定成 功路线: 美国的 STEM 教育战略》, 标志着 STEM 教育 在美国进入了一个新阶段 ${ }^{[5]}$ 。英国注重 STEM 政策的 顶层设计, 确立了国家级 STEM 示范活动, 并启动了 国家科学技术大赛和高等教育 STEM 计划 ${ }^{[6]}$ 。新加坡 也已经普及 STEAM 综合教学的模式, 并取得系列成果 [7]

我国的 STEM 教育起步较晚, 2014 年作为教育综 合改革的试点项目, 上海成立了 “STEM+研究中心”。 2015 年，教育部颁布的《关于 “十三五” 期间全面 深入推进教育信息化工作的指导意见 (征求意见稿)》 标志着我国深入开展 STEM 教育。2018 年, 中国教育 科学研究院发布《中国 STEM 教育 2029 行动计划》, 呼吁更多的社会力量协同开展 STEM 教育。同年, 国 家教育部、工业和信息化部与中国工程院等部门提出 要培养跨学科、跨专业的工程人才。2020 年 5 月教 育部提出探索学科复合交叉合作规律, 探索科技人才 培养的新模式。

\section{STEM 教育的主要特征}

STEM 教育是在科技飞速发展与人才需求发生重 大变化的形势下涌现出的一种教育理念, 它高度重视 科学、技术、工程与数学等多学科的融合, 突出培养 学生的动手能力与创新意识, 综合文献 ${ }^{[6-10]}$, STEM 教 育的主要特征有:

（1）学科融合性。每个专业的知识结构都有局限, STEM 教育主张从真实问题出发, 将不同学科有机结 合起来对某些复杂问题形成更为全面的一般性认识, 着力培养学生的创新实践能力。朱莉. 汤普森. 克莱因 根据课程融合程度将跨学科课程分为多学科课程、交 叉学科课程和超学科课程三个层次, 目前 STEM 教育 的课程形式主要在交叉学科课程层次, 能够融合原有 各学科目标, 完成学科重构 [8]。也就是说, STEM 教 育提倡打破科学、技术、工程和数学等学科间的壁垒, 从多学科知识融合应用的角度提高学生解决实际问 题的能力。

(2) 问题的社会性。STEM 强调问题源于生活, 要 求学生结合社会实际问题进行实践, 用学科融合的思 维方式解决社会实际问题 [8], 每年都有不少地方政
府、企事业单位发布技术攻关项目，其中好多问题往 往需要学生到企事业单位甚至生产一线学习、调查, 才能全面认识问题、抓住问题的本质乃至找到合适的 解决方法。

(3) 问题驱动式学习。学生根据问题储备知识与 技能是 STEM 教育的重要环节。学生在认识与解决问 题的过程中可以形成 手脑并用与边实践边学习的局 面, 科学素养与工程思维得到提升。

(4) 趣味性学习。兴趣是学习动力的源泉, 大学 生喜欢具有专业背景或时代特征的问题, 这类以专业 知识为主, 紧密联系实际生活的问题更容易激发学生 的学习兴趣, 使学生在学习中体会到问题的趣味性 ${ }^{[9]}$ 。

（5）技术性。STEM 教育重视运用专业技术、数理 思维、建模与计算方法，使用相关技术分析问题、制 定解决方案。因此 STEM 教育需要学生把技术作为认 知工具、利用技术重新建构问题、运用技术解决问题。

\section{STEM 教育的开展形式}

STEM 教育要求学生根据问题或项目背景组成小 组, 建立协作分工与开放共享的混合式学习环境, 目 前国内外大学 STEM 教育的开展形式主要有:

第一种是课程形式。目前, 美国、英国、土耳其、 爱尔兰等不少国家的高校已经开设了 STEM 课程, 我 国一流大学大多都进行了通识教育改革, 不少地方高 校实施了文理渗透, 开设数学模型与实践课等 ${ }^{[11]}$ 。

目前, 以跨学科课程实施 STEM 教育比较适合我国学 生的整体水平, 数据表明跨学科学习的效果比传统的 学科学习好很多 ${ }^{[12]}$ 。这种形式的优点是容易制定课程 教学大纲、课程简介、教学计划、考核方式与学习目 标, 教师可及时提供学习支持和学习资源。随着学生 学习模式的完善与师生互动的增多, 可采取超学科形 式的 STEM 教育 ${ }^{[13,14]}$ 。

第二种是项目形式。学生根据知识背景, 项目主 要分为学科项目与问题项目, 后者对项目成员的要求 更高, 学生的自主权扩大, 教师的职责主要是宏观指 导 ${ }^{[15]}$ 。目前, 项目在 STEM 教学中的比例逐渐提高, 问题项目也愈加受到重视。以项目形式开展 STEM 教 育的优势主要有: (1) 转变学生的学习方式, 从注重 专业知识的学习转向基于学科融合分析实际问题的 学习, 形成综合学习与实践学习并存的局面; (2) 以 项目为出发点, 推动应用取向、创新取向的学习, 可 提高学生的实践水平与协作能力; (3) 真实问题可开 阔学生的认知能力, 激发其创新意识, 实现 STEM 教 育分工合作、共同创造的教育目标。

此外, 学校的 STEM 教育资源有限, 需要研究机 构、企业等社会各界提供更多的学习平台。美国已制 定了各界联合实施 STEM 教育的政策, 也有不少大学 联合企业开展 STEM 教育, 我国实施的卓越工程师教 育培养计划与新工科建设都有利于开展 STEM 教育 ${ }^{[14]}$ 。 


\section{4. 我校大学数学教学的探索与实践}

近代科学技术的发展很大程度上得益于微积分 的创立, 许多问题可以归结为基于专业知识的数学问 题。STEM 教育提倡科学、技术、工程和数学等学科 的大融合, 数学是掌握科学、技术和工程设计的工具, 我校是一所以工科为主的地方高校, 它不仅重视工程 与技术教育内容, 还强调学科教学的配套整体设计。 为适应融入学校工程教育认证、卓越工程师培养计划 以及新工科建设与发展, 针对当前地方高校数学创新 能力培养中普遍存在的问题, 受前述 STEM 教育的主 要特征与开展形式的启示, 我校大学数学教学基于 STEM 教育理念做了一些有效的探索与实践。

\section{1. 积极探索和实践基于课程与项目融合形 式的 STEM 教育}

我校大学数学教学从课程与项目两方面, 积极探 索和实践 STEM 教育与课程专业融合。一方面, 开设 《微积分》《微积分晋阶》《线性代数》和《概率论与 数理统计》等公共基础课程, 《基于 MATLAB 的高等数 学实验》、《凸优化模型、算法与应用》、《面向 MCM\&ICM 的数学实验与数学建模》等创新实践课程以及《传染 病动力学建模与仿真实验》等创新实验课程, 构建科 学的面向数学创新能力培养的立体化课程体系; 另一 方面, 以全国大学生数学建模竞赛、美国大学生数学 建模竞赛等为驱动, 以教师科研和自选研究为依托, 以国家级、市级和校级创新创业训练计划项目为抓手, 提高学生参与创新创业的积极性和主动性。具体举措:

(1) 在课堂中增加 STEM 教育元素。这种可以给学 生联想与思考的空间, 比如引入导数的概念时, 尽量 挖掘具有真实意境的问题, 如赛跑中的速度与爆发力, 让学生建立变化率的概念, 并让学生联想类似案例。 再如矩阵的乘法教学及时引入连接图及其矩阵表示, 可布置具有 STEM 特征的问题作为课后作业。例如比 赛排名问题, 现有 5 个队 (编号为 1-5) 参加单循环 比赛, 它们的比赛结果为: 1 队胜 $2 、 3$ 队; 2 队胜 3 、 $4 、 5$ 队； 4 队胜 $1 、 3 、 5$ 队； 5 队胜 $1 、 3$ 队, 试为这 5 个队排名次 ${ }^{[16]}$. 假定其排名规则为: 按获胜的次数 排名次, 若两队胜的次数相同, 则按直接胜与间接胜 的次数之和排名次。

首先, 考虑连接图及其邻接矩阵, 比如 1 队胜 2

队, 用结点 1 到结点 2 的有向线段表示, 其他场 次可类似表示, 结果如图 1 ,

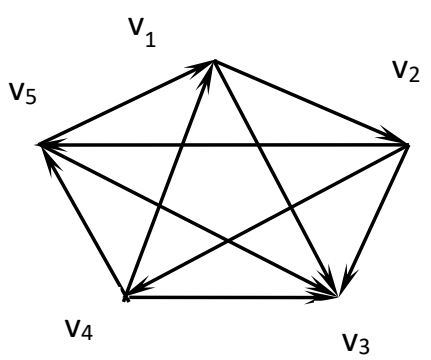

图 1. 5 个队之间的 胜负关系.

Fig.1. The winning and losing relationship among the 5 teams.

其邻接矩阵为

$$
M=\left(\begin{array}{lllll}
0 & 1 & 1 & 0 & 0 \\
0 & 0 & 1 & 1 & 1 \\
0 & 0 & 0 & 0 & 0 \\
1 & 0 & 1 & 0 & 1 \\
1 & 0 & 1 & 0 & 0
\end{array}\right) .
$$

其次, 矩阵 $M$ 的行和最大值 3 出现在第 2 与第 4 行, 表示 2 队与 4 队直接胜 3 场, 冠军属于他们之 一。同时第 1 行与第 5 行的行和为 2 , 表明 1 队与 5 队均胜了 2 场, 它们应该在 3、4 名, 区分 2 队与 4 队, 或 1 队与 5 队的具体名次需要考虑间接胜的场次, 即矩阵 $M^{2}$ 的行和。由 $M+M^{2}$ 行和分别为 5、8、0、 $7 、 4$, 最终排名为 $2 、 4 、 1 、 5 、 3$. 如果还有行和相 等, 可借助经由 $k-1$ 个队伍的间接胜 (即路径长为 $k$ ) 的场次, 即矩阵 $M^{k}(k=3,4,5)$ 的行和来区分。

上面问题还可以对间接胜赋予权重进行研究, 也 可以研究交通、社会网络等问题。学生只有大胆思考、 大胆尝试, 才能更快成长。STEM 教育理念下的大学 数学教学, 离不开大量的实践探索。首先, 要选好真 实案例, 以问题为导向, 基于科学原理和基本知识引 导学生寻找问题的解决方案; 其次, 运用数学知识建 模与分析, 借助计算机或相关技术解决问题; 最后, 以小组讨论、分析的形式开展 STEM 课堂教学可以调 动学生积极性, 教学效果也比较好。

(2) 开设数学文化与学科交叉课程选修课。当 前文科与理科的鸿沟导致高校培养的人才已经越来 越不能适应当今自然科学与社会科学高度渗透的现 代化社会, 数学文化无疑是沟通二者的桥梁。文科学 生通过数学文化的学习可以初步了解数学概貌, 而理 科生在接受大学数学的专业训练外, 亦可获得人文学 科的培养。再有受课堂教学学时的限制, 大学数学系 列课程中具有重要应用的拉格朗日乘子法、最小二乘 法等一些应用性强的知识不能在课堂详细讲解。为此, 我们在考虑文理之间差异以及实际应用问题的需要, 
通过开设《数学文化与创新思维》等通识课程和《凸 优化模型、算法与应用》等实践课程来弥补现有教学 内容和学时不足的弊端, 以此激发学生学数学、用数 学和爱数学的兴趣。

(3) 以各类各级竞赛为驱动, 利用第二课堂讲 授具有交叉学科背景的创新型课题和项目。比如数学 建模竞赛、市场调查大赛、互联网+大学生创新创业 大赛、大学数创新训练计划项目等都需要学生的团队 协作, 通常 3-5 名学生一组。尽管单个学生面对 STEM 项目非常感兴趣, 但是限于知识储备、学科以及时间 等限制而不能很好的完成竞赛或项目。在大学数学教 学外, 开设《面向 MCM\&ICM 的数学实验与数学建模》 等创新实践课程以及《传染病动力学建模与仿真实验》 等创新实验课程, 无疑有利于提高学生的学科交叉的 认知与实践能力。

\section{2. 积极探索和实践产教融合、产研融合的 STEM 教育}

交叉学科是创新的源泉。当前, 我国把交叉学科 提升到前所未有的高度。近年来, 学院围绕 “开设哪 些交叉学科课程, 怎么建设交叉学科课程, 如何提升 交叉学科的教师队伍” 等进行初步探索, 大学数学以 此为契机进行了产教融合、产研融合的 STEM 教育教 学改革与实践。

(1) 学校层面为大学数学产教融合产研融合的 STEM 教育教学改革提供可能并创造条件。我校是工 学、经济、管理、艺术、设计等多学科互相渗透、协 调发展的全日制普通高等学校, 是教育部 “卓越工程 师教育培养计划” 首批试点高校、全国地方高校新工 科建设牵头单位、上海市 “高水平地方应用型高校” 试点建设单位。在学校这个层次上具有了科学、技术、 工程、数学与艺术等学科的框架。为了提高学生的综 合能力, 学院结合数学教师具备的数学、统计学、金 融学、计算机科学与技术和管理科学与工程等学科背 景, 融导师-研究生-本科生学习和研究于一体, 着力 构建创新文化与科研反哺的互融互通体系, 为大学数 学教学组建 STEM 教师队伍与开展 STEM 教育奠定了基 础。

(2) 大学数学紧密结合学生的兴趣与专业进行产 教融合的 STEM 教育教学改革与实践。为低年级与高 年级学生均开设选修课, 诸如《数学文化与创新思维》, 《数学实验与数学建模》《基于 MATLAB 的高等数学实 验》等课程。这类课程涉及的专业背景比较多, 根据 教师的专业与特长, 组织多位教师分模块授课, 有效 提升了课程教学质量。为了加强过程管理和质量控制, 每门课程都制定了教学大纲、授课要求, 以及发布开 放性作业, 以提高学生的实践能力、促进学生的全面 发展, 从而完善交叉学科课程。后续尝试组建不同学 院乃至不同学校的教师开设的选修课, 建立一条高效、 高度跨专业的合作道路。学校聘请优秀企业界工程技 术人员做学业导师、兼职导师, 也鼓励学科交叉教师
到企业中多调研、多实践，根据社会企业的需求，建 设交叉学科教师队伍, 这些举措推动交叉学科与产业 界的合作, 有利于构建开放的交叉学科学习平台, 有 利于健全交叉学科建设的形式。

(3) 大学数学倡导科研反哺教学, “学以致用”与 “用以致学” 并进, 进行产研融合的 STEM 教育教学 改革与实践。学院成立校级智能计算与应用统计研究 中心, 聚焦智能计算与应用、应用统计与优化及数学 基础理论三个学科前沿方向组建三个优秀科研团队, 团队负责人主持在研国家自然科学基金面上项目、军 委科技委前沿创新计划重点项目子课题和全国统计 科学研究一般项目等重要课题, 多名教师获得上海市 自然科学奖和上海市科技进步奖。依托骨干教学团队 和科研团队, 一批中青年教师快速成长为学科带头人 和学科骨干, 科研成果丰硕, 科研反哺教学; 以此为 基础, 实施骨干教师、学术方向带头人和学科带头人, 都需要指导大学生创新创业训练, 指导学科竞赛, 教 师成长的过程, 始终伴随着创新创业能力和素质的提 升; 把学生创新精神、创业意识和创新创业能力作为 STEM 教育培养质量评价的重要指标, 和企事业单位 进行合作, 以学院与雷根集团、金仕达校企合作基地、 联合攻关人工智能科技发展基金等产学研基地和项 目为依托, 深入推动产研融合协同育人。

\section{5. 结论与讨论}

教育的现状表明传统的教学模式传授经典知识 的效率较高, 但学生的自主学习、解决实际问题与创 新实践能力的表现不理想。STEM 教育鼓励学生探索 真实问题、整合多学科知识与选择更合理的技术方法, 可以激发学生的学习兴趣, 可以提高他们多学科间的 沟通能力, 从而提高他们的创新实践能力。从传统的 教学模式到 STEM 教育模式的转变, 学生也从经典知 识的积累转变为运用知识、创造新知识、新方法, 从 而转变为综合能力的提高。

基于 STEM 教育理念, 我校大学数学教学在课程 与项目融合、产教融合和产研融合等方面做了积极的 探索与实践, 有效提高了学生数学创新实践与思维等 综合素养, 助力学校推动 “卓越工程师人才计划” 以 及新工科建设。大学数学课程的理论性较强, 培养学 生的运算能力、空间想象能力和逻辑推理能力离不开 课堂教学; 另一方面, 现在的教学模式需要改革, 以 提高学生应用数学的能力, 即解决专业问题或 STEM 专业问题的能力。大学数学教学除了在授课时创设 STEM 问题场景、课堂改编一些数学建模赛题作为课 后作业, 还需要学校之间以及学校与企业、研究机构 合作开发一些 STEM 课程, 或者设立更多的项目, 比 如大学生创新项目、企业或政府发布的项目, 更多的 产教融合、产研融合项目才能促进学生的全面发展。

STEM 教育已对大学数学革新教育模式, 完善人 才培养方案等方面产生明显的推动作用, 后续我校大 学数学教学将基于 STEM 教育理念加强改革与实践的 
广度与深度: 进一步培训教师的跨学科教学能力; 结 合学生的综合素质开展 STEM 教育, 让学生尽快形成 主动探索的学习观念; STEM 教育教学上需要更多的 教学设施与资源, 寻求争取学校、政府企业的更多投 入等。

\section{项目基金}

本文为上海市教委重点课程建设项目 “微积分 A” (项目编号: 371)、高等学校大学 数学教学研究与发展中心 2020 年教学改革项 目（项目编号：CMC20200413）和上海工程技 术大学教育科学研究项目 (项目编号: x202121001）的阶段性成果之一。

\section{REFERENCES}

[1] Zhu, X,Y., Kong, H.B. (2008) On the Development of Human Resources of Science and Technology. Research in Higher Education of Engineering, 2008(2):21-25 (In Chinese).

[2] American Council on Education. (2006) Increasing the Success of Minority Students in Science and Technology. American Council on Education, Washington.

[3] Morrison J.S. (2006) Attributes of stem education: the students, the academy, the classroom, Ties STEM Education Monograph Series. Baltimore Teaching Institute for Excellence in STEM, Maryland.

[4] PCAST. (2012) PCAST, Engage to Excel: Producing One Million Additional College Graduates with Degrees in Science, Technology, Engineering, and Mathematics; Executive Office of the President: Washington, DC, USA.

[5] Xue, D., Wu, J.X. (2021) The Latest Core Competencies of American Graduate STEM Education: Policy Context, Standard Framework and Training Path. Journal of Graduate Education, 62(2): 90-97 (In Chinese).

[6] Xu, Y.X., Tan, Q.J., Shao, J. (2021) The Enlightenment of Mathematics Major Construction of the Open University of England to China's Open Education. Journal of Taiyuan City Vocational College, (3):91-93 (In Chinese).
[7] Li, Y., Li, T. (2019) The Applied Learning Programme of Singapore and Its Enlightenments for China's Basic Education. Journal of Kashi University. 40(2):109-116 (In Chinese).

[8] Bai, Y.X. (2016) The Trend of STEM Education Innovation in the United States: for Fair and High-quality Learning Experience. Research in Higher Education of Engineering, 2016(6): 171179 (In Chinese).

[9] Yu, S.Q., Hu, x. (2015) STEM Education and Its Model for Interdisciplinary Integration. Open Education Research. 21(4):13-22 (In Chinese).

[10] Zhang, F. (2020) STEM: a profound impact on the reform and development of basic education Concept and practice. Journal of Shanghai Educational Research, (4): 5-11 (In Chinese).

[11] David M. Rubush, Kari L. Stone. (2020) A Learning Community Involving Collaborative Course-Based Research Experiences for Foundational Chemistry Laboratories. Education Sciences Vol:10(4), 117:1-11

[12] Zhao, H.C., Zhang, N.Y., Ma, J.W. (2020) Interdisciplinary Learning Community for STEM Education: Promoting Learning Paradigm Shift. Open Education Research, 26(3):91-98 (In Chinese).

[13] Hu, Y., Jiang, Q. (2019) The opportunities and challenges of integration of Mathematics Education and STEM (STEAM) Education - International Symposium on Mathematics Education and STEM (STEAM) Education. Journal of Mathematics Education, 28(6):92-94 (In Chinese).

[14] Mo, J.F., Huang, H., Yang, L.P. (2020) The Construction of STEM Courses in the Emerging Engineering Education: Theories, Experiences and Tactics. Renmin University of China Education Journal, (4):11-22 (In Chinese).

[15] Dong,Y., H, J.Y. (2019) Research on the Application of Project-based Learning in University Teaching. Modern Educational Technology. 29(9): 53-58(In Chinese).

[16] Ju, Y.M. (2002) Linear Algebra. Tsinghua University Press, Beijing (In Chinese). 